\title{
Efficacy of serum miRNA test as a non- invasive method to diagnose nonalcoholic steatohepatitis: a systematic review and meta-analysis
}

Shengliang Xin, Qiao Zhan, Xiaofan Chen, Jinghang Xu and Yanyan Yu*

\begin{abstract}
Background: Nonalcoholic steatohepatitis (NASH) is a key turning point during the progression of nonalcoholic fatty liver disease (NAFLD). Recent studies have shown that serum miRNA tests may be effective in the diagnosis of NAFLD. We conducted a meta-analysis to assess the evidence for the diagnostic efficacy of serum miRNAs in patients with NAFLD and its subtype, NASH, in particular.

Methods: After a systematic review, sensitivity, specificity, and area under the receiver operating characteristics curve (AUROC) were pooled to determine the efficacy of serum miRNA test for the diagnosis of NAFLD and NASH. Clinical utility was evaluated by Fagan's nomogram and likelihood ratio scattergram. Heterogeneity was evaluated by subgroup analysis and meta-regression. Publication bias was detected by Deeks' funnel plot.

Results: We included 27 trials containing 1775 NAFLD patients (including simple steatosis and NASH) and 586 NASH patients. For NAFLD vs NASH, the pooled sensitivity, specificity, and AUROC were (0.71 vs. 0.74$)$, (0.76 vs. 0.85 ) and (0.80 vs. 0.86), respectively. Serum miRNA had high accuracy for distinguishing NASH from simple steatosis, with an AUROC of 0.91. Among the most commonly studied serum miRNAs, miRNA-34a showed moderate diagnostic accuracy for NAFLD and the lowest heterogeneity (sensitivity $P^{2}=5.73 \%$, specificity $P^{2}=33.16 \%$, AUROC $=$ 0.85). According to subgroup analysis and meta-regression, a lower BMl $\left(<30 \mathrm{~kg} / \mathrm{m}^{2}\right)$ might be a crucial source of heterogeneity.

Conclusions: As a novel non-invasive method, serum miRNA test exhibited robust diagnostic efficacy for NASH. Among these well-studied miRNAs, miRNA-34a was more available for diagnosis. Diagnosis of NAFLD by serum miRNA is more likely to be accurate in patients with $B M l \geq 30 \mathrm{~kg} / \mathrm{m}^{2}$.
\end{abstract}

Keywords: MicroRNA, Nonalcoholic steatohepatitis, Obesity, Body mass index

* Correspondence: Yuyanyan010@163.com

Department of Infectious Diseases, Peking University First Hospital, Beijing,

China

C C The Author(s). 2020 Open Access This article is licensed under a Creative Commons Attribution 4.0 International License, which permits use, sharing, adaptation, distribution and reproduction in any medium or format, as long as you give appropriate credit to the original author(s) and the source, provide a link to the Creative Commons licence, and indicate if changes were made. The images or other third party material in this article are included in the article's Creative Commons licence, unless indicated otherwise in a credit line to the material. If material is not included in the article's Creative Commons licence and your intended use is not permitted by statutory regulation or exceeds the permitted use, you will need to obtain permission directly from the copyright holder. To view a copy of this licence, visit http://creativecommons.org/licenses/by/4.0/. The Creative Commons Public Domain Dedication waiver (http://creativecommons.org/publicdomain/zero/1.0/) applies to the data made available in this article, unless otherwise stated in a credit line to the data. 


\section{Background}

Non-alcoholic fatty liver disease (NAFLD) has become a major burden among chronic liver diseases. According to the latest epidemiological studies, the prevalence of NAFLD is approximately $25 \%$ worldwide [1]. In developed countries such as the United States, the prevalence of NAFLD is $30 \%$ [1]. In developing countries such as China, the prevalence has reached up to $32.9 \%$ [2]. NAFLD comprises a spectrum of pathological conditions, including simple steatosis (NAFL), nonalcoholic steatohepatitis (NASH), fibrosis, cirrhosis and hepatocellular carcinoma (HCC). It should be noted that NASH is a crucial stage during NAFLD progression. Studies have shown that approximately one-sixth of NAFL patients progress to NASH [1], and $20 \%$ of NASH patients can develop cirrhosis [3]. Furthermore, some studies have indicated that NASH patients have a $60 \%$ greater chance of progression to HCC than that of NAFL patients [4]. The traditional view suggests that HCC formation is a multi-stage process, involving inflammation, fibrosis and cirrhosis. However, recent research found that NASH can progress to HCC without fibrosis and cirrhosis [5]. Therefore, early diagnosis of NAFLD, in particular $\mathrm{NASH}$, is important.

Liver biopsy is the gold standard for diagnosis and staging of NASH; however, its clinical application is restricted by patients' reluctance. Moreover, liver biopsy has potential complications such as bleeding, especially when patients are in decompensated conditions. Therefore, there is an urgent need to develop a non-invasive approach for diagnosis of NASH. To date, the wellstudied serological biomarker is cytokeratin (CK)-18. CK-18 is an indicator of hepatocyte apoptosis. Increased CK-18 suggests severe damage in the liver parenchyma [6]. In terms of diagnostic efficacy for NASH, CK-18 shows moderate accuracy with specificity of $80 \%$ and the area under the receiver operating characteristics curve (AUROC) of 0.83 , however, its sensitivity is low at $60 \%$ [7]. Other indexes such as alanine aminotransferase (ALT) and aspartate aminotransferase (AST) can give false-negatives [8]. Thus, a better non-invasive biomarker for diagnosing NASH is still needed.

MicroRNAs (miRNAs) are short, non-coding singlestranded RNAs strand of 20-25 nucleotides. miRNAs play complicated and important roles in regulating the expression of downstream genes [9]. Many experiments and clinical trials have confirmed that miRNAs are closely related to NAFLD [10-13]. miRNAs target a variety of genes related to lipid metabolism and proinflammatory factors, that are involved in pathogenesis of NAFLD [14]. A recent meta-analysis reported that circulating miRNA has moderate diagnostic accuracy in NAFLD [15]. However, NAFLD is a general term that consists of several pathological subtypes. Simple steatosis, fibrosis and cirrhosis can all be identified by alternative methods such as B-scan ultrasonography, computed tomography (CT) and Fibroscan [16-18]. NASH is the most hazardous stage, but cannot be diagnosed by radiological methods. Hence, there is a need to evaluate the diagnostic efficacy of serum miRNA for NASH. In this meta-analysis, we analyzed the value of serum miRNAs for diagnosis of NAFLD, in particular the subtype, NASH.

\section{Methods}

\section{Literature retrieval}

We have registered a review protocol on the website https://www.crd.york.ac.uk/prospero

(CRD42020172385), and performed this study based on the preferred reporting items for systematic reviews and meta-analysis (PRISMA) guidelines (Additional file 1: PRISMA 2009 Checklist). All relevant articles on the application of miRNA in the diagnosis of NAFLD available on multiple electronic databases including PubMed, Science Direct, and Cochrane Library up to February 1, 2020 were searched. The retrieval strategy was ("NAFLD" OR "Non-alcoholic Fatty Liver Disease" OR "NASH" OR "Non-alcoholic Steatohepatitis") AND ("microRNAs" OR "miRNA" OR "microRNA" OR "miR" OR "hsa-miR"). Language was not limited. Additional literature was not included in the study. We collected 3956 records according to retrieval strategy. The preliminary screening process (title and abstract screening) was performed by two authors (SLX and QZ) independently and blindly. The second screening process (full-text review) was performed by all authors. The literature was managed by Endnote X9.

\section{Eligibility criteria}

We included articles that met the following conditions: (1) diagnostic trials; (2) trials with control and case cohorts (NAFLD or NASH); and (3) all NAFLD cases (including NAFLD and NASH) were confirmed by liver biopsy. We excluded articles with the following conditions: (1) other irrelevant liver diseases (e.g., alcoholic liver disease, viral hepatitis and drug-induced liver injury); (2) miRNAs were extracted from other tissues than serum; (3) necessary statistical data were not available for calculating the test performance parameters including true-positive (TP), false-positive (FP), true-negative (TN) and false-negative (FN) rates; and (4) duplicated records.

\section{Common characteristics of included trials}

(1) NAFLD was confirmed when $>5 \%$ of hepatocytes developed steatosis; (2) NASH was confirmed by NAFLD activity score (NAS) $\geq 5$; (3) miRNA level was quantified by reverse transcription-polymerase chain reaction (RTPCR); (4) in NAFLD trials, the control cohort comprised 
healthy individuals, and the case cohort comprised NAFLD patients (including NAFL and NASH); and (5) in NASH trials, the control cohort comprised individuals with NAS $<5$, and the case cohort comprised NASH patients with NAS $\geq 5$.

\section{Data extraction and literature quality assessment}

We constructed a $2 \times 2$ contingency table and calculated the numbers of TP, FP, FN and TN results in each trial. Quality assessment of the included articles was assessed by two authors independently using the Quality Assessment of Diagnostic Accuracy Studies (QUADAS-2), as previously described [18].

\section{Statistical analysis}

Stata SE version 15 was used to perform the metaanalysis. The first step was to calculate pooled statistical values, including sensitivity, specificity, positive likelihood ratio (PLR), negative likelihood ratio (NLR) and diagnostic odds ratio (DOR). $I^{2}$ statistic was used to evaluate the heterogeneity among trials. $I^{2}>50 \%$ indicated a considerable heterogeneity, thus, random-effects model was used. The second step was to conduct summary receiver operating characteristics (SROC) curve analysis. If SROC curve had a "shoulder-arm shape", the threshold effect was considered. AUROC values of 0.5$0.7,0.7-0.9$ and $0.9-1.0$ suggest low, moderate and high diagnostic accuracy, respectively. The third step was to detect the source of heterogeneity by conducting subgroup analysis and meta-regression. For meta-regression, a covariate with $P<0.05$ was considered the significant factor to induce heterogeneity. The fourth step was to assess publication bias. Deeks' funnel plot was applied to examine the potential publication bias caused by any asymmetry of the trials. $P<0.05$ for the slope coefficient indicated test asymmetry and suggested significant publication bias [19]. Finally, we used Fagan's nomogram and a likelihood ratio scattergram to evaluate the clinical utility of this method.

\section{Results}

Literature retrieval, characteristics and quality assessment The literature search yielded 3956 records. Using Endnote X9, duplicate records $(n=295)$ and non-target article types $(n=636)$ were excluded. During the preliminary screening process (title and abstract screening), we eliminated irrelevant articles $(n=988)$ and experimental studies $(n=1967)$. During the second screening process (full-text review), we included nine articles that met the eligibility criteria [20-28]. A flow diagram of the literature selection process is presented in Fig. 1.

The meta-analysis was conducted on 27 trials included in nine articles. We extracted the necessary characteristics (e.g., geographic region, target miRNA, the mode of
miRNA regulation, disease type, sample size, BMI, proportion of males and diagnostic sensitivity and specificity) of each trial. Trials were categorized by these factors as follows: (1) geographic region: Asian $(n=11)$, non-Asian $(n=16)$; (2) regulation mode: miRNA in upregulation mode $(n=21)$, in downregulation mode $(n=$ 6); (3) disease type: NASH $(n=13)$, NAFLD (including NAFL and NASH) $(n=14) ;(4)$ average BMI: $\geq 30 \mathrm{~kg} / \mathrm{m}^{2}$ $(n=15),<30 \mathrm{~kg} / \mathrm{m}^{2}(n=9)$; and (5) proportion of males: $\geq 50 \%(n=12),<50 \%(n=8)$. Detailed information of these trials is presented in Additional file 1: Table S1.

The overall sample size (including controls and cases) in this meta-analysis was 4036, of which 2764 were in NAFLD trials and 1272 in NASH trials (Fig. 2a). Moreover, we summarized the target miRNAs among these 27 trials, and the top 4 in terms of total sample size were miR-122 $(n=1107)$, miRNA-99a $(n=792)$, miRNA-34a $(n=642)$ and miRNA panel $(n=368)$ (Fig. 2b). We conducted a Cochrane bias graph to assess the quality of each included article according to the QUADAS-2 tool (Additional file 1: Figure S1).

\section{Diagnostic value of serum miRNAs in NAFLD cases Pooling all trials to evaluate the diagnostic efficacy of serum miRNA for total NAFLD}

Significant heterogeneity existed among the trials based on the sensitivity and specificity values (pooled $I^{2}$ for sensitivity and specificity was 94.82 and $88.37 \%$, respectively). Hence, we used the random-effects model in our study. The pooled values were as follows: sensitivity $=0.72[95 \%$ confidence interval (CI): 0.64-0.79], specificity $=0.81(95 \%$ CI: 0.75-0.86), PLR = 3.77 (95\% CI: 2.86-4.96), NLR = 0.34 (95\% CI: 0.27-0.44), DOR = 10.95 (95\% CI: 7.0517.01), and AUROC $=0.84$ (95\% CI: 0.80-0.87) (Fig. 3). These results indicated that serum miRNA had moderate diagnostic accuracy for total NAFLD.

Next, we evaluated efficacy of the most studied serum miRNAs, namely, miRNA-122, miRNA-99a and miRNA-34a, for the diagnosis of total NAFLD. (1) The pooled values of miRNA-122 were as follows: sensitivity $=0.84(95 \%$ CI: $0.77-0.90) I^{2}=80.62 \%$; specificity $=$ 0.72 (95\% CI: 0.61-0.81) $I^{2}=85.44 \%$; PLR $=3.01(95 \%$ CI: 2.12-4.27); NLR = 0.22 (95\% CI: 0.14-0.33); DOR = 13.79 (95\% CI: 7.29-26.06); and AUROC $=0.86(95 \%$ CI: 0.82-0.89) (Additional file 1: Figure S2). (2) The pooled values of miRNA-99a were as follows: sensitivity $=0.82(95 \%$ CI: $0.71-0.89) I^{2}=93.46 \%$; specificity $=$ 0.82 (95\% CI: 0.53-0.95) $I^{2}=96.90 \% ; \quad P L R=4.58(95 \%$ CI: $1.30-16.12) ; \quad \mathrm{NLR}=0.22 \quad(95 \% \quad \mathrm{CI}: \quad 0.11-0.47)$; $\mathrm{DOR}=20.42(95 \% \mathrm{CI}: 2.86-146.00) ;$ and $\mathrm{AUROC}=$ 0.87 (95\% CI: 0.84-0.90) (Additional file 1:Figure S3). (3) The pooled values of miRNA-34a were as follows: sensitivity $=0.81$ (95\% CI: 0.76-0.85) $I^{2}=5.73 \%$; specificity $=0.83 \quad(95 \%$ CI: $0.77-0.87) \quad I^{2}=33.16 \% ; \quad$ PLR $=4.70$ 


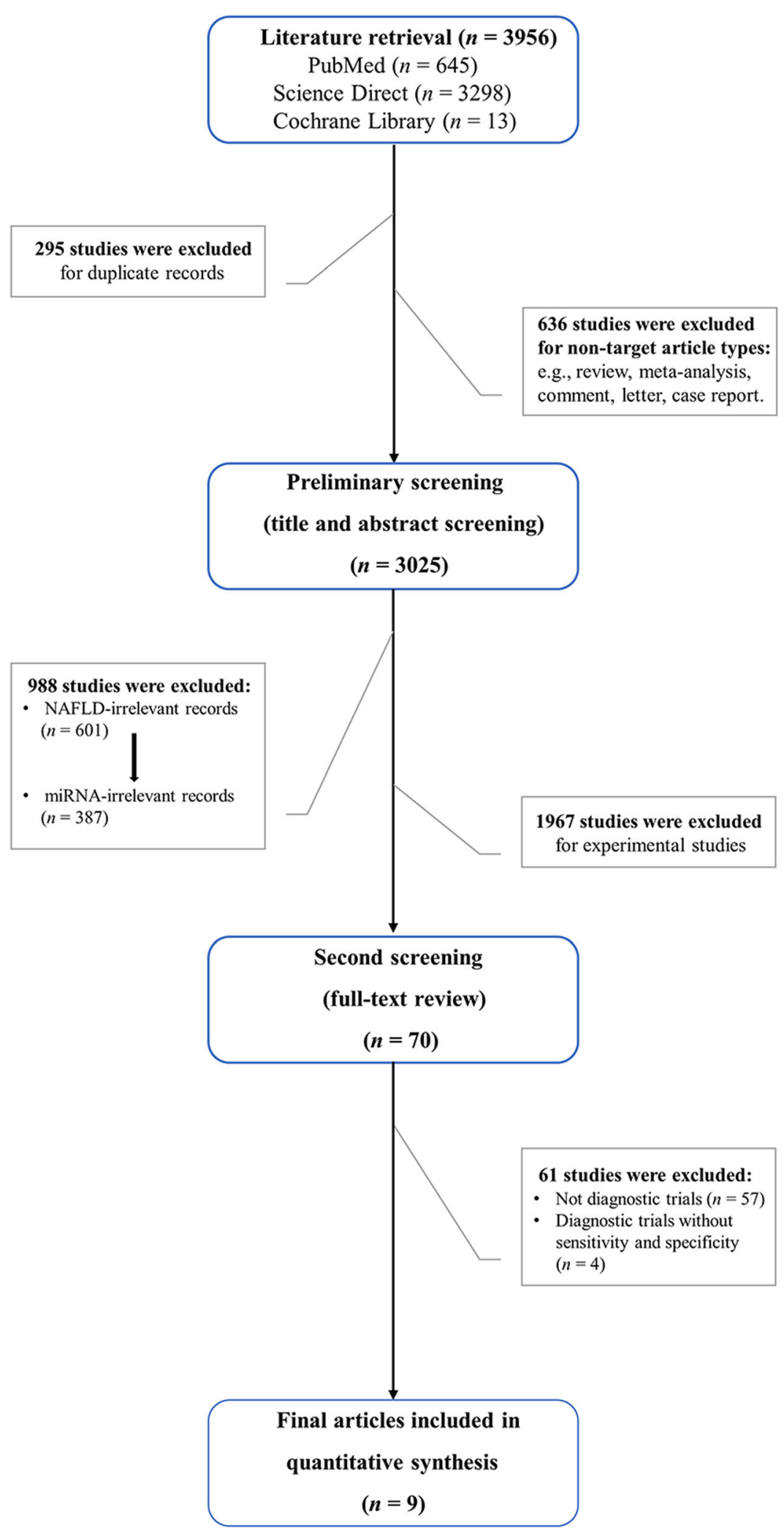

Fig. 1 Article selection process 


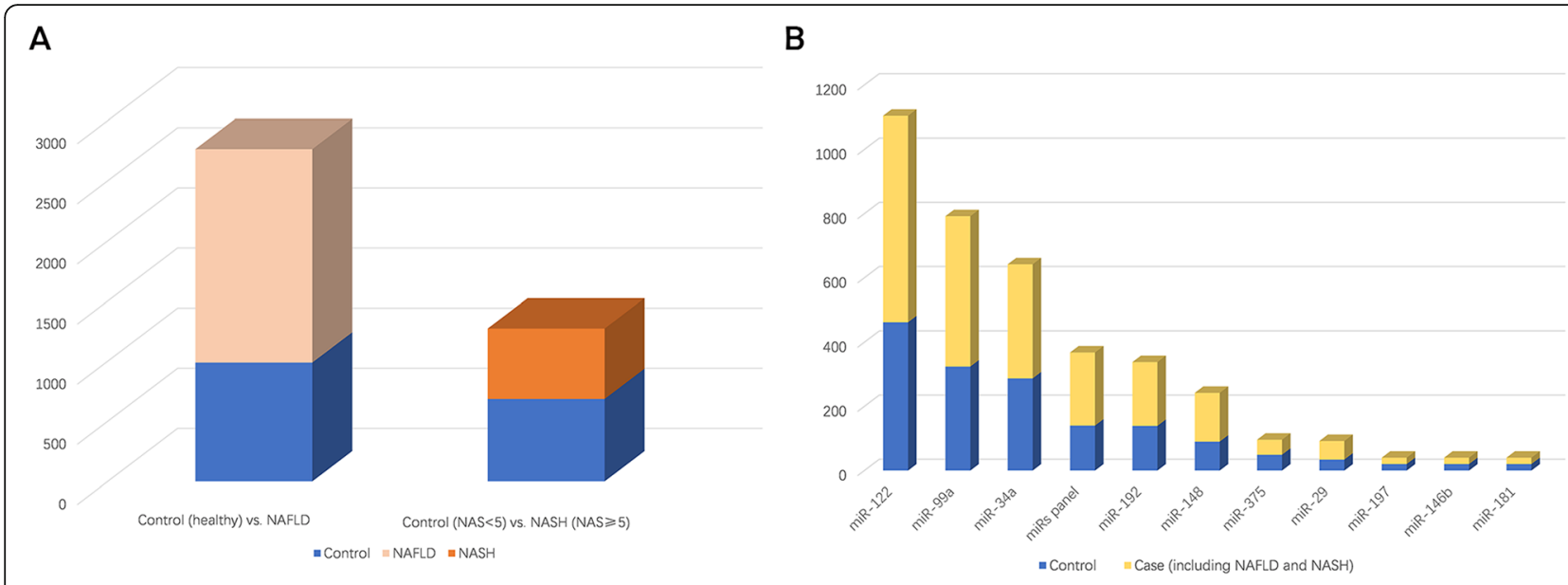

Fig. 2 a: Total sample size in NAFLD trials and NASH trials. b: Total sample size of each target serum miRNA

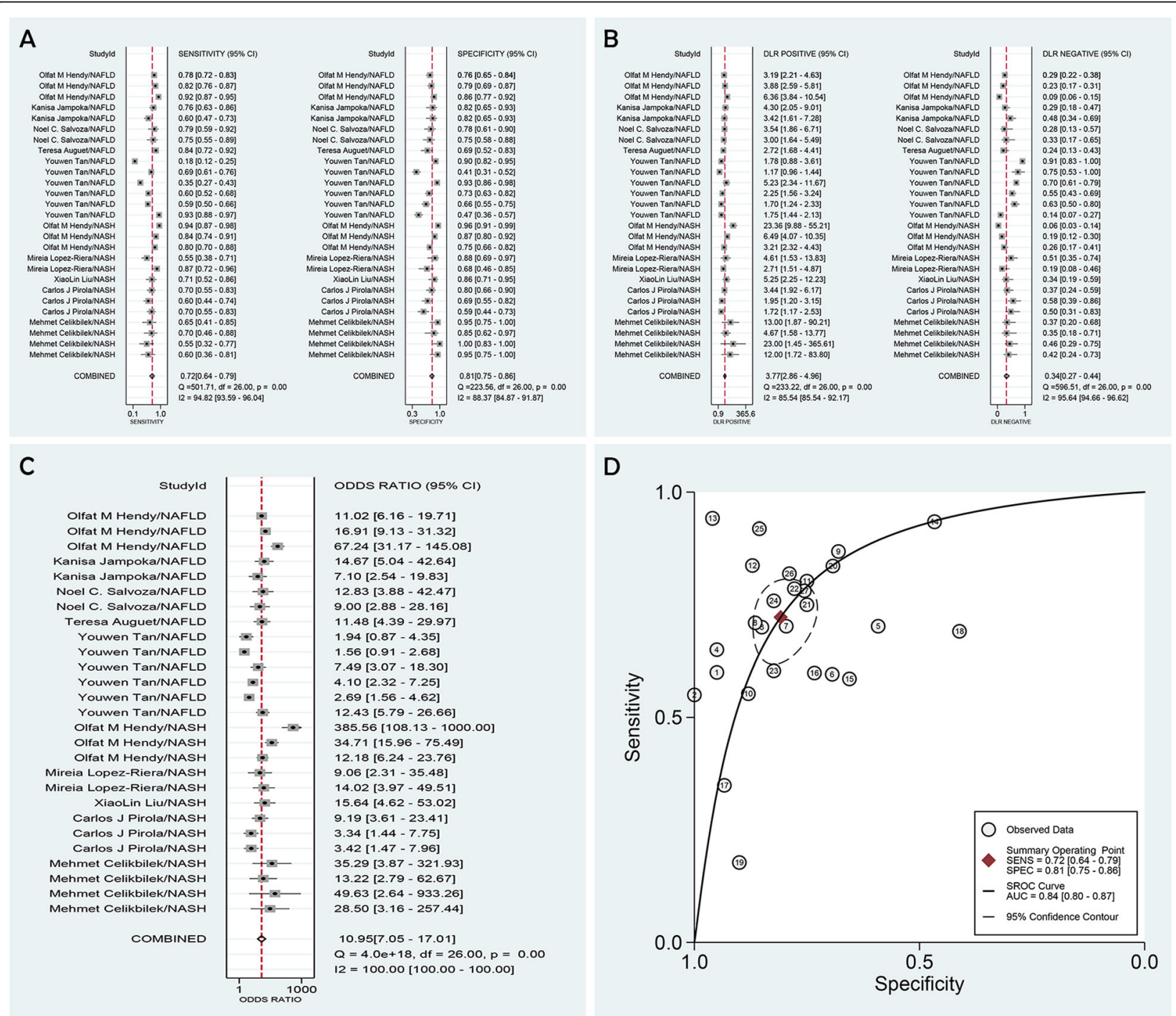

Fig. 3 Forest plots and meta-analysis of trials showing pooled sensitivity and specificity (a), PLR and NLR (b) and DOR (c) of serum miRNA for diagnosis of total NAFLD (case vs. control). d: SROC curve of serum miRNA for diagnosis of total NAFLD (case vs. control) 
(95\% CI: 3.51-6.30); NLR $=0.23$ (95\% CI: $0.18-0.29$ ); $\mathrm{DOR}=20.34(95 \% \mathrm{CI}: 13.08-31.60)$; and $\mathrm{AUROC}=0.85$ (95\% CI: 0.82-0.88) (Additional file 1: Figure S4). These results indicated all three miRNAs had a moderate diagnostic accuracy for total NAFLD. It should be noted that, miRNA-34a showed the lowest heterogeneity; thus, miRNA-34a was more available to diagnose total NAFLD.

\section{Pooling trials N0.1-13 and trials N0.14-27 separately to evaluate the diagnostic efficacy of serum miRNA for NASH and NAFLD}

(1) The pooled values of NASH trials were as follows: sensitivity $=0.74(95 \% \mathrm{CI}: 0.66-0.81) I^{2}=74.94 \%$; specificity $=0.85$ (95\% CI: $0.77-0.91) I^{2}=79.60 \%$; $\mathrm{PLR}=5.01$ (95\% CI: 3.11-8.05); NLR $=0.31$ (95\% CI: 0.23-0.42); DOR = 16.24 (95\% CI: 8.17-32.28); and AUROC $=0.86$ (95\% CI: 0.83-0.89) (Additional file 1: Figure S5).

(2) The pooled values of NAFLD trials were as follows: sensitivity $=0.71(95 \%$ CI: $0.58-0.81) I^{2}=96.88 \%$; specificity $=0.76(95 \%$ CI: $0.68-0.83) I^{2}=90.57 \%$; $\mathrm{PLR}=2.99$ (95\% CI: 2.24-3.99); NLR $=0.38$ (95\% CI: 0.26-0.55); DOR = 7.93 (95\% CI: 4.66-13.49); and $\mathrm{AUROC}=0.80$ (95\% CI: 0.77-0.84) (Additional file 1: Figure S6).

These results revealed that serum miRNA had moderate diagnostic accuracy in both NASH and NAFLD trials. Moreover, serum miRNA showed a better diagnostic efficacy for NASH than that for NAFLD, as indicated by the higher DOR, higher AUROC, and lower heterogeneity.

\section{Pooling trials NO.9-13 to evaluate the diagnostic efficacy of serum miRNA for distinguishing NASH from NAFL}

The pooled values for these trials were as follows: sensitivity $=0.83$ (95\% CI: 0.70-0.91) $I^{2}=86.22 \%$; specificity $=$ 0.85 (95\% CI: 0.74-0.92) $I^{2}=85.11 \%$; and AUROC $=0.91$ (95\% CI: 0.88-0.93). These results suggested that serum miRNA had high accuracy for discriminating NASH from NAFL (Additional file 1: Figure S7).

\section{Subgroup analysis}

To investigate the source of heterogeneity, we conducted subgroup analysis (Qualitative Research). Trials NO.127 were divided into subgroups based on five study factors: geographic region, type of disease, regulation mode, proportion of males and BMI. Subsequently, we calculated pooled values for each subgroup (Table 1). The results as follows.

(1) Geographic region: compared with Asian trials, nonAsian trials had higher sensitivity (0.77 vs. 0.64) and specificity (0.83 vs. 0.76 ) and significantly lower heterogeneity (sensitivity $I^{2} 81.33 \%$ vs. $96.08 \%$, specificity $I^{2} 76.03 \%$ vs. $\left.92.08 \%\right)$. Non-Asian trials had a higher DOR (17 vs. 6) and AUROC (0.87 vs. 0.77).

(2) Type of disease: These results were described in Section 2.2.

(3) Regulation mode: compared with upregulation trials, downregulation trials had lower sensitivity (0.66 vs. 0.74 ), higher specificity ( 0.89 vs. 0.78 ), and lower heterogeneity (sensitivity $I^{2} 59.82 \%$ vs. $95.92 \%$, specificity $I^{2} 58.57 \%$ vs. $\left.90.07 \%\right)$. Apart from that, downregulation trials had a higher DOR (16 vs. 10) but equivalent AUROC values (0.83 vs. 0.83).

(4) Proportion of males: compared with trials that had a proportion of males $\geq 50 \%$, trials with a proportion of males $<50 \%$ had higher sensitivity (0.77 vs.0.64) and specificity ( 0.87 vs. 0.74 ) and significantly lower heterogeneity (sensitivity $I^{2} 81.17 \%$ vs. $95.58 \%$, specificity $I^{2} 58.46 \%$ vs. $\left.90.78 \%\right)$. Trials with a proportion of males $<50 \%$ had a higher DOR (21 vs. 5 ) and AUROC (0.89 vs. 0.75).

(5) BMI: compared with trials with $\mathrm{BMI}<30 \mathrm{~kg} / \mathrm{m}^{2}$, trials with $B M I \geq 30 \mathrm{~kg} / \mathrm{m}^{2}$ had higher sensitivity (0.77 vs. 0.63$)$ and specificity (0.84 vs. 0.75$)$ and significantly lower heterogeneity (sensitivity $I^{2}$ $82.34 \%$ vs. $96.69 \%$, specificity $I^{2} 76.67 \%$ vs. $\left.93.11 \%\right)$. Trials with $\mathrm{BMI} \geq 30 \mathrm{~kg} / \mathrm{m}^{2}$ had a higher DOR (17 vs. 5) and AUROC (0.87 vs. 0.76).

Collectively, serum miRNA showed more accurate diagnosis of total NAFLD in these conditions: nonAsian, presence of $\mathrm{NASH}$, female predominance and $\mathrm{BMI} \geq 30 \mathrm{~kg} / \mathrm{m}^{2}$. In terms of heterogeneity, all of the above five study factors could be the potential sources.

\section{Meta-regression}

To determine the source of heterogeneity, we performed meta-regression (Quantitative Research). Geographic region, type of disease, miRNA regulation mode and miRNA profiling were considered as covariates. Due to lack of some data, proportion of males and BMI were not included in this meta-regression. We made the assignment as follows: disease $(\mathrm{Yes}=\mathrm{NASH}, \mathrm{No}=\mathrm{NAFLD})$, regulation mode (Yes = upregulation, No = downregulation), region (Yes $=$ Asian, No $=$ Non-Asian $),$ and miRNA profiling $($ Yes $=$ single miRNA, No $=$ miRNA panel $)$. The reason for heterogeneity might be related to geographic region (Asian) (sensitivity $P<0.01$, specificity $P<0.001$ ), but was unrelated to type of disease, miRNA regulation mode and miRNA profiling (Fig. 4). Thus, Asian trials could be the significant factor to induce heterogeneity.

The major difference between Asian and non-Asian trials was in BMI. Of the 16 Non-Asian trials, 15 had a $\mathrm{BMI} \geq 30 \mathrm{~kg} / \mathrm{m}^{2}$ and one did not provide the BMI. Of the 11 Asian trials, nine had a BMI $<30 \mathrm{~kg} / \mathrm{m}^{2}$ and two did 


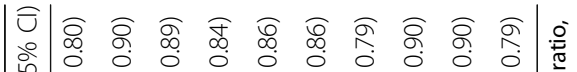

的

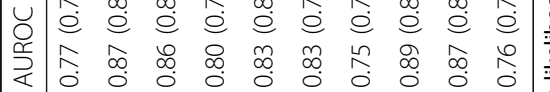

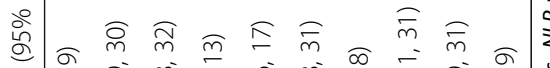

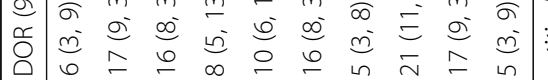

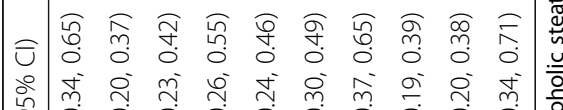

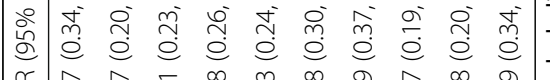

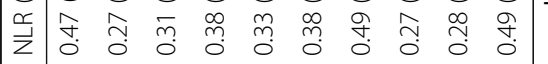

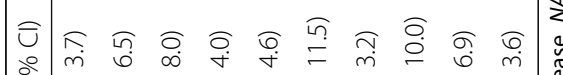

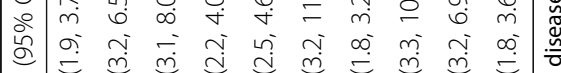

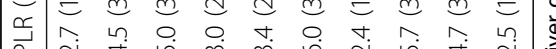

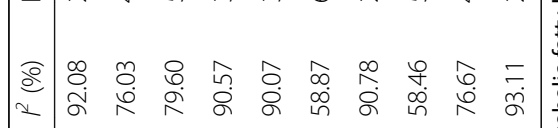

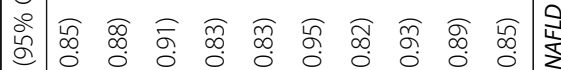

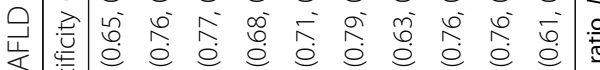




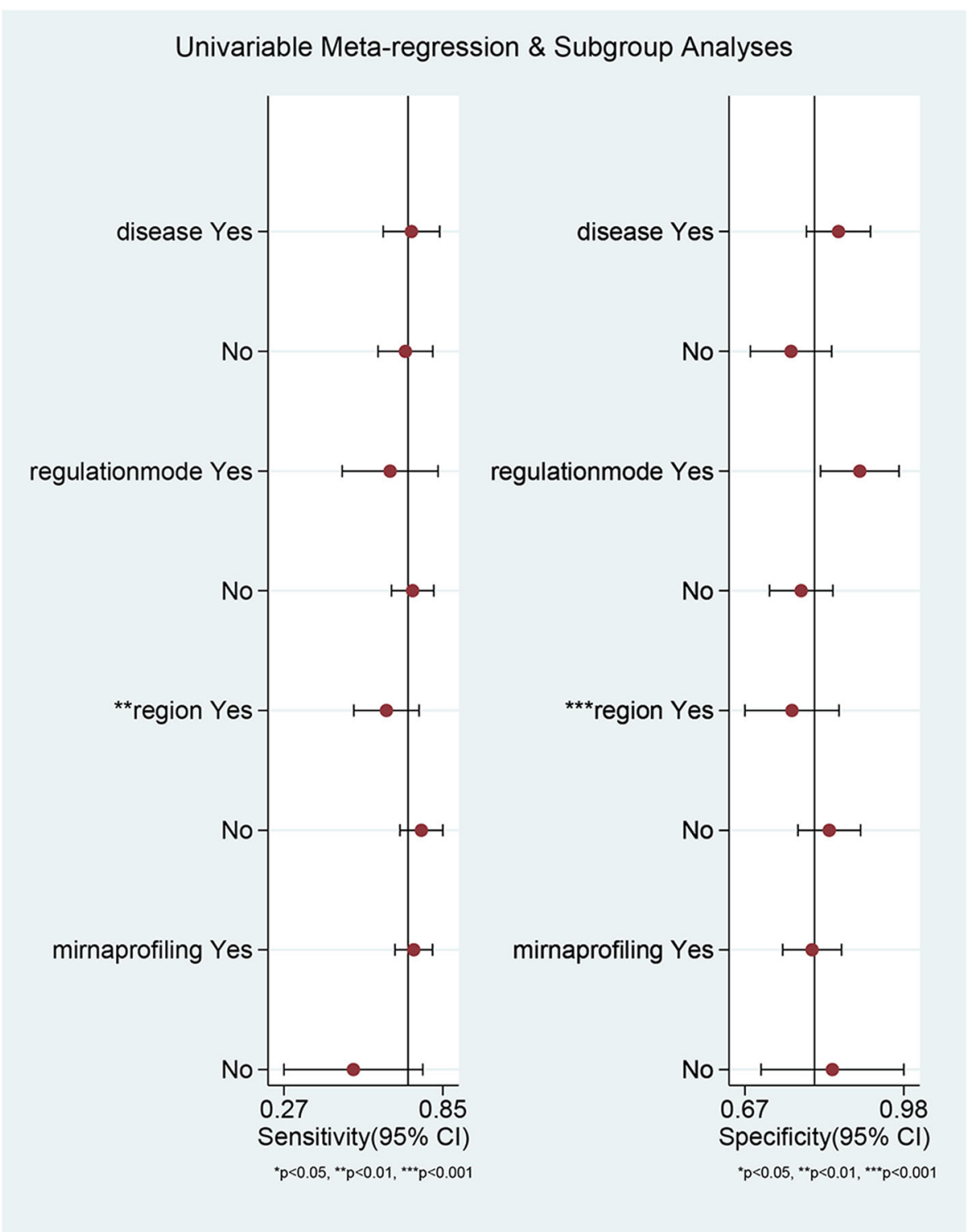

Fig. 4 Meta-regression analysis of serum miRNA for diagnosis of total NAFLD (case vs. control). The assignment was made as follows: disease $($ Yes $=\mathrm{NASH}, \mathrm{No}=\mathrm{NAFLD})$, regulation mode (Yes = upregulation, $\mathrm{No}=$ downregulation), geographic region (Yes = Asian, No = Non-Asian), and miRNA profiling (Yes $=$ single miRNA, No $=$ miRNA panel)

not provide the BMI. Moreover, the pooled values of subgroups categorized by geographic region or BMI were similar. Apart from that, no significant differences in other aspects were seen between Asian and non-Asian trials. Therefore, based on these findings, we speculated that BMI $<30 \mathrm{~kg} / \mathrm{m}^{2}$ could be a potential predominant factor to induce heterogeneity rather than Asian region. After omitting trials with study factors $\mathrm{BMI}<30 \mathrm{~kg} / \mathrm{m}^{2}$, proportion of males $\geq 50 \%$, NAFLD, and miRNA in upregulation mode, the heterogeneity of sensitivity and specificity showed a downward trend: sensitivity $I^{2} 94.82 \%$ vs. $81.28 \%$ vs. $80.39 \%$ vs. $0 \%$, specificity $I^{2} 88.37 \%$ vs. $72.92 \%$ vs. $76.92 \%$ vs. $25.99 \%$ (Additional file 1: Table S2).

\section{Clinical utility}

We used Fagan's nomogram to examine NASH trials (NO. 1-13) and NAFLD trials (NO. 14-27 (Fig. 5a-b). Pre-test probability was set at $50 \%$. The results were as follows. (1) Among NASH trials, the PLR was 5 accompanied by post-test probability of $83 \%$ and NLR was 0.31 accompanied by post-test probability of $24 \%$; and (2) Among NAFLD trials, the PLR was 3 accompanied by post-test probability of $75 \%$ and NLR was 0.38 accompanied by post-test probability of $27 \%$. These findings revealed that serum miRNA exhibited higher positive diagnostic value for NASH than that for NAFLD. Furthermore, a likelihood ratio scattergram was constructed 

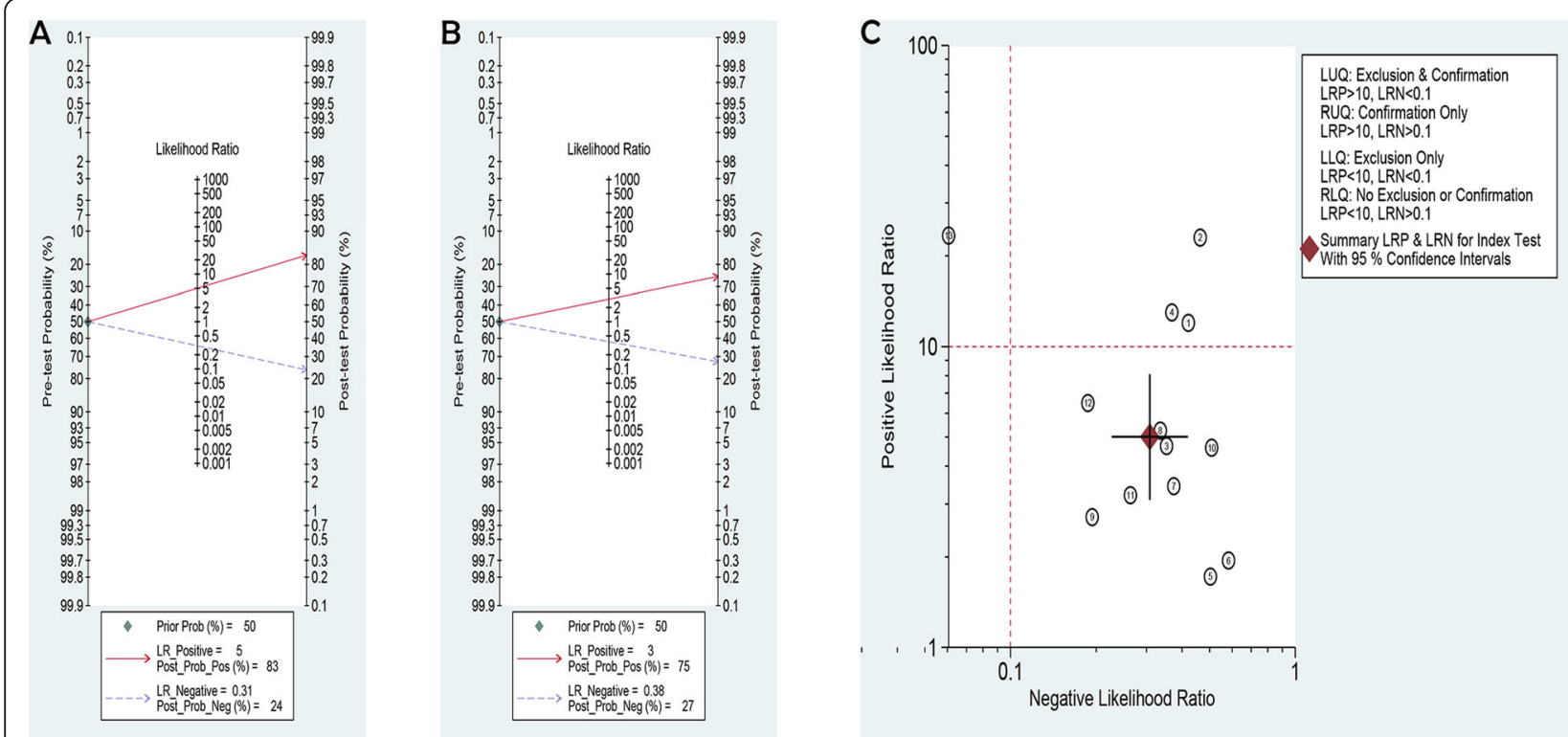

Fig. 5 a: Fagan's nomogram of serum miRNA for diagnosis of NASH (NAS $\geq 5$ vs. < ). Pre-test probability $=50 \%$ (patients with moderate suspicion for NASH), the post-test positive and negative probability of NASH were 83 and 24\%, respectively. $\mathbf{b}$ : Fagan's nomogram of serum miRNA for diagnosis of NAFLD (NAFLD vs. healthy control). Pre-test probability $=50 \%$ (patients with the moderate suspicion for NAFLD), the posttest positive and negative probability of NAFLD were 75 and 27\%, respectively. c: The likelihood ratio scattergram of serum miRNA for diagnosis of NASH (NAS $\geq 5$ vs. <5). LRP: positive likelihood ratio; LRN: negative likelihood ratio; LUQ: left upper quadrant; RUQ: right upper quadrant; LLQ: left lower quadrant; RLQ: right lower quadrant

for NASH trials (Fig. 5c). The summary likelihood ratio for serum miRNA test was located in the lower right quadrant, which meant serum miRNA test did not reach the pathological standard for exclusion and confirmation, and hence, its clinical utility was limited.

\section{Publication bias}

Based on Deeks' funnel plot (Fig. 6a-c), publication bias was not observed in the trials in which serum miRNA was used to diagnose total NAFLD $(P=0.77)$, NAFLD $(P=0.84)$ and NASH $(P=0.29)$. In addition, publication bias was not observed that when serum miRNA-34a was used to diagnose total NAFLD $(P=0.46)$ (Fig. 6d).

\section{Discussion}

In this study, we systematically reviewed the diagnostic value of serum miRNA for NAFLD. Different from previous studies, we focused on NASH, which is a subtype of NAFLD. Our meta-analysis was performed on 27 trials containing 1775 NAFLD patients (including NAFL and NASH) and 586 NASH patients. We compared the diagnostic efficacy among trials from two perspectives: first, we compared the use of the most studied serum miRNAs (i.e., miRNA-122, miRNA-99 and miRNA-34a) to diagnose total NAFLD; second, we compared the use of serum miRNAs to diagnose total NAFLD, NAFLD and NASH. In addition, we detected the sources of heterogeneity by pooling data from multiple factors, including geographic region, type of disease, miRNA regulation mode, proportion of males, BMI and miRNA profiling.

The major three conclusions of this study were as follows (1) Serum miRNA showed a higher diagnostic efficacy for NASH than that for total NAFLD and NAFLD. Notably, serum miRNA had high accuracy in distinguishing NASH from NAFL. (2) Among the most commonly studies serum miRNAs, miRNA-34a showed the most stable efficacy, with moderate diagnostic accuracy for total NAFLD. (3) A BMI $<30 \mathrm{~kg} / \mathrm{m}^{2}$ may be the predominant reason for heterogeneity. NAFLD patients with obesity $\left(\geq 30 \mathrm{~kg} / \mathrm{m}^{2}\right.$ ) were more likely to have accurate diagnosis by serum miRNA test.

\section{First, we still need better non-invasive methods for diagnosis of NASH}

NAFLD is the most common liver disease in the world. It is estimated that by 2030 , the number of NAFLD patients in the United States will reach 100 million. Its subtype, NASH, accounts for 7-30\% of NAFLD patients [1]. In Asia, the prevalence of NASH seems to be higher. One study found that $63.5 \%$ of liver biopsies from NAFLD patients were confirmed to be NASH [29]. $\mathrm{NASH}$ is an important turning point for the progression to end-stage liver disease. Therefore, the early and accurate diagnosis of NASH is needed. The gold standard 
A

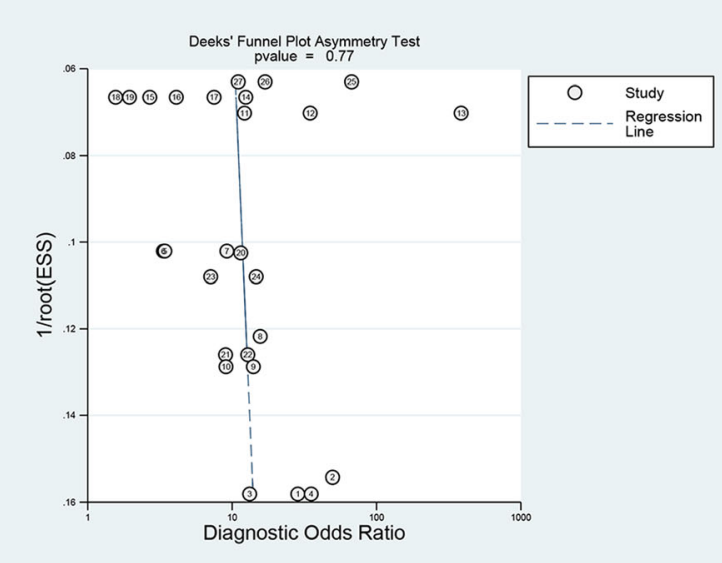

C

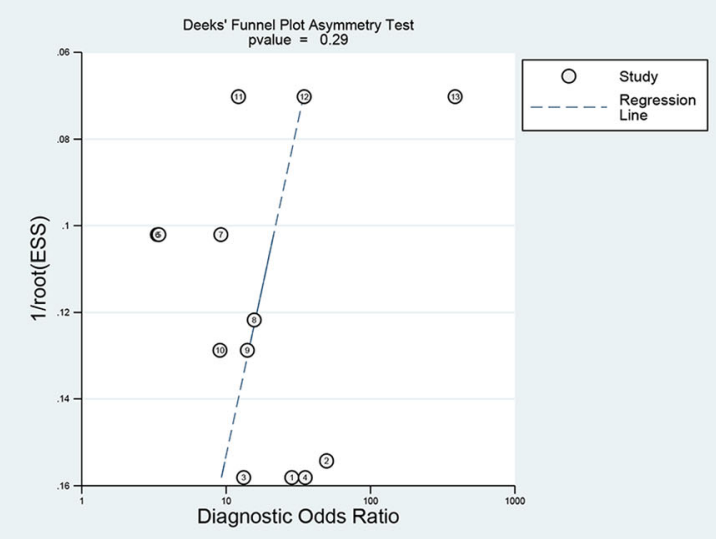

B

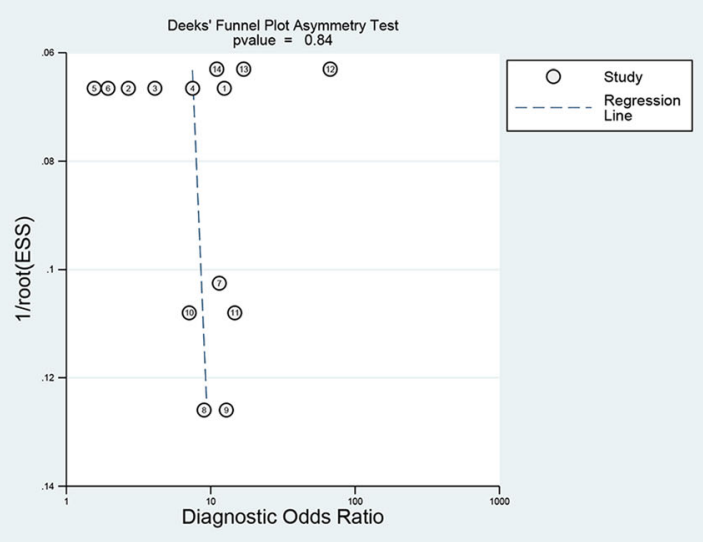

D

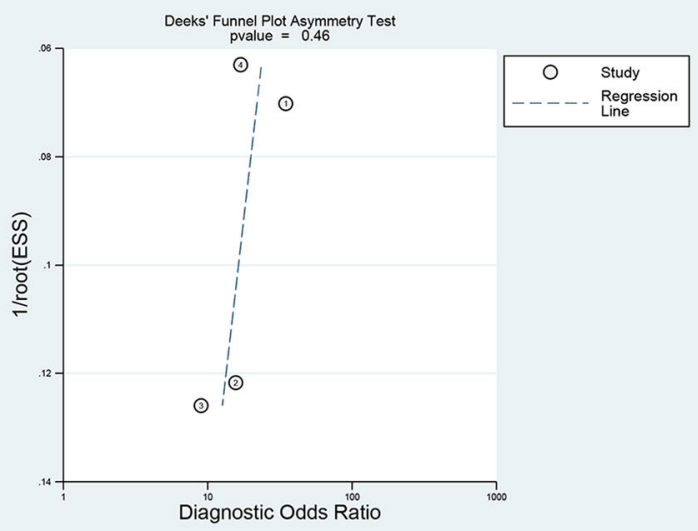

Fig. 6 Estimation of the publication bias by Deeks' funnel plots for all involved trials (a), NAFLD trials (b), NASH trials (c) and miRNA-34a trials (d)

for diagnosis and staging of NASH is liver biopsy. However, biopsies cannot be widely used due to complications and patients' reluctance [30]. Therefore, noninvasive diagnostic tools are necessary. However, up to now, satisfactory biomarkers have not been identified. (1) Liver function parameters such as AST and ALT, can reflect damage of hepatocytes, but this depends on severity of NASH. Some NASH patients might present with normal levels of AST and ALT [31]. (2) CK-18 is a common index for diagnosis of NASH, because it reflects necrosis and apoptosis of hepatocytes. CK-18 is characterized by good specificity but weak sensitivity. To enhance the sensitivity of this biomarker, it needs to be combined with other indexes [7, 32-35]. (3) Inflammation indexes such as interleukin-6 and tumor necrosis factor- $\alpha$, always show poor specificity [36]. (4) At present, it is reported that comprehensive scoring systems (e.g., NashTest and ActiTest) have moderate efficacy for the diagnosis of NASH, but involve excessive indexes and are costly [37]. Therefore, we still need better non-invasive methods for diagnosis of NASH.

\section{Second, serum miRNA has a higher diagnostic accuracy} for NASH than that for NAFLD

Recent studies have revealed the close relationship between miRNA and NAFLD [38-40]. miRNAs are noncoding RNAs of 20-25 nucleotides. They have complex regulatory mechanisms. In brief, miRNAs can suppress or promote expression of target genes [41]. miRNAs widely participate in multiple pathological processes of NAFLD [14, 41], and their serum levels differ significantly between healthy individuals and NAFLD patients. Hence, they have become a new potential non-invasive biomarker for diagnosis of NAFLD. We found that serum miRNAs had moderate diagnostic accuracy for NAFLD, as reported by Cai, et al. [15]. The difference is that our subgroup analysis showed that serum miRNA has a higher diagnostic accuracy for NASH than that for NAFLD: the pooled values of NAFLD trials vs. NASH trials were sensitivity ( 0.71 vs. 0.74$)$, specificity ( 0.76 vs. 0.85 ), and AUROC ( 0.80 vs. 0.86 ). It has been found that serum levels of miRNA gradually increase or decrease between healthy individuals, NAFL patients to NASH patients, which indicates disease deterioration [23, 42, 43]. 
That could be one reason for serum miRNAs exhibiting the better accuracy for diagnosis of NASH. Moreover, we found a high accuracy of serum miRNA for distinguishing NASH from NAFL with AUROC of 0.91 . Similarly, a recent study reported that miRNA-34a had moderate accuracy for distinguishing between NASH and NAFL (AUROC $=0.78$ ) [44]. Our study involved a total of 14 miRNAs, which have several roles in the pathogenesis of NAFLD [14, 45], such as, lipid synthesis (miRNA-122), fatty acid $\beta$-oxidation (miRNA-34a, 122), endoplasmic reticulum stress (miRNA-30, -34a, -122), inflammation (miRNA-34a, -99a, -146b), fibrosis (miRNA-122), tumorigenesis (miRNA-99a), and cell autophagy and apoptosis (miRNA-34a). The relationships between miRNAs and NASH pathogenesis can be both one-to-many and many-to-one. Therefore, different from traditional non-invasive diagnostic methods, serum miRNA can reflect "all-aspects" of NASH, which contributes to its diagnostic accuracy for NASH.

\section{Third, serum miRNA-34a is an available index to diagnose NAFLD}

We focused on three well-studied miRNAs (miRNA-122, -99a, and -34a). All of them had moderate accuracy for diagnosing total NAFLD and miRNA-34a showed the lowest heterogeneity. For miRNA-122 vs. miRNA-99a vs. miRNA-34a, the pooled values were: sensitivity $I^{2}(80.62 \%$ vs. $93.46 \%$ vs. $5.73 \%)$, specificity $I^{2}(85.44 \%$ vs. $96.90 \%$ vs. $33.16 \%$ ), and AUROC (0.86 vs. 0.87 vs. 0.85 ). miRNA-34a exhibited the most stable efficacy among the three miRNAs. Therefore, serum miRNA-34a is a more available biomarker to diagnose NAFLD. Previous studies have shown that miRNA-34a plays pivotal roles in NAFLD progression. (1) In terms of lipid metabolism, miRNA-34a can downregulate the PPAR $\alpha$ signaling pathway. PPAR $\alpha$ is a key transcription factor of fatty acid oxidation that facilitates the transfer of fatty acids into the mitochondria for oxidation. On the contrary, blocking the PPAR $\alpha$ signaling pathway could induce lipid accumulation in hepatocytes [46]. (2) In addition, PPAR $\alpha$ is involved in liver inflammation by activating Kupffer cells [47]. (3) In terms of apoptosis, by repressing sirtuin-1, miRNA-34a increases p53 acetylation and transcription, leading to induction of pro-apoptotic genes such as PUMA, and finally, apoptosis [48]. Collectively, miRNA-34a moderates the "first hit" (abnormal lipid metabolism) and "second hit" (inflammation and apoptosis) for the pathogenesis of NAFLD. In other words, miRNA34a could reflect the entire course of NAFLD, including onset and subsequent progression. Therefore, that is one reason for miRNA-34a exhibiting more stable diagnostic efficacy than other miRNAs. We did not evaluate the diagnostic efficacy of miRNA-34a for NASH separately. A previous study has found that miRNA-34a can distinguish NASH from NAFL with an AUROC of 0.78 [44].

\section{Fourth, the diagnostic efficacy of serum miRNA for NAFLD may depend on BMI}

In subgroup analysis, miRNA showed greater accuracy for diagnosing NAFLD in patients with $B M I \geq 30 \mathrm{~kg} /$ $\mathrm{m}^{2}$. The pooled values of trials with $\mathrm{BMI} \geq 30 \mathrm{~kg} / \mathrm{m}^{2}$ and $<30 \mathrm{~kg} / \mathrm{m}^{2}$ were: sensitivity (0.77 vs.0.63), specificity (0.84 vs. 0.75$)$, and AUROC (0.87 vs. 0.76). In meta-regression, the covariate, geographic region (Asian), demonstrated significant differences in sensitivity and specificity. Thus, Asian region could be a significant factor for heterogeneity. Moreover, in view of the obvious overlap when trials were separately categorized by geographic region and BMI, we speculated that BMI $<30 \mathrm{~kg} / \mathrm{m}^{2}$ was the potential predominant factor for heterogeneity rather than Asian region. When we omitted the trials with $\mathrm{BMI}<30 \mathrm{~kg} /$ $\mathrm{m}^{2}$, heterogeneity of sensitivity and specificity were mitigated: sensitivity $I^{2} 94.82 \%$ vs. $81.28 \%$, specificity $I^{2} 88.37 \%$ vs. $72.92 \%$. In our study, Asian patients with NAFLD were characterized by lower BMIs, which ranged from 24 to $28 \mathrm{~kg} / \mathrm{m}^{2}$. A recent epidemiological investigation supports our findings: the prevalence of NAFLD in people with BMI $<25 \mathrm{~kg} / \mathrm{m}^{2}$ was $8-20 \%$ in China, $7 \%$ in India, $15 \%$ in Korea and $13 \%$ in Japan. Lean or non-obese NAFLD has become a new trend in Asia [49]. The pathogenesis of lean NAFLD and obese NAFLD are not entirely consistent. Studies have found that PNPLA3 polymorphism plays a specific role in NAFLD in the non-obese population $[50,51]$. Considering this peculiarity, we do not recommend the application of serum miRNA test for diagnosis in NAFLD patients with $B M I<30 \mathrm{~kg} / \mathrm{m}^{2}$. Collectively, the diagnostic efficacy of serum miRNA for NAFLD may depend on BMI. Due to lack of some data, we did not directly evaluate the effect of BMI on heterogeneity by meta-regression. Thus, it needs to be confirmed in a further study.

\section{Fifth, the serum miRNA test could be used for the early diagnosis of NASH in clinical practice}

(1) In general, serum miRNA showed moderate efficacy for diagnosis of NASH. In terms of clinical utility, Fagan's nomogram revealed that when a patient was in a dilemma about diagnosis or exclusion of $\mathrm{NASH}$, miRNA could provide an effective reference: if a positive result was acquired, the patient would have $83 \%$ probability of confirmation by liver biopsy; If a negative result was acquired, the patient would has $76 \%$ probability of exclusion by liver biopsy. (2) Individually, because of the necessity for early diagnosis of NASH in clinical practice, sensitivity deserves special attention. Our study suggests that serum miRNA shows robust positive efficacy for diagnosis of NASH: $\mathrm{NASH}$ trials had higher pooled sensitivity $(0.74$ vs. 
0.71), PLR (5.01 vs. 2.99), $\operatorname{DOR}(16.24$ vs. 7.93$)$, and post-test positive probability ( $83 \%$ vs. $75 \%$ ) than those of NAFLD trials; pooled sensitivity was further increased to 0.83 when distinguishing $\mathrm{NASH}$ from NAFL; likelihood ratio scattergram indicated that more trials $(n=4$ vs. 1$)$ reached the pathological confirmation standard (positive likelihood ratio, LRP > 10) rather than pathological exclusion standard (negative likelihood ratio, LRN $<0.1$ ). (3) The serum miRNA test has a limited clinical utility. In the likelihood ratio scattergram, the summary LRP \& LRN for serum miRNA test was located in the right lower quadrant, which means that serum miRNA test still has less in clinical utility than the gold standard, liver biopsy. Therefore, we should improve this test in the future as follows: the diagnostic flow of serum miRNA should be standardized, including sampling and testing; dynamic monitoring should be performed; this method should be applied mostly among obese individuals $\left(B M I \geq 30 \mathrm{~kg} / \mathrm{m}^{2}\right)$; this method should be combined with other non-invasive methods, such as CK18 , which has a good specificity but a weak sensitivity, to enhance the overall diagnostic efficacy. In summary, there are still few accurate and non-invasive diagnostic methods that can replace liver biopsy. As a novel and effective method, the serum miRNA test is quantitative in real-time and easy to conduct. When suspected NASH patients present with normal liver function parameters, an early serum miRNA test should be considered.

\section{Last, there are still some limitations to this study}

First, the cutoff value was an important cause of heterogeneity; however, most trials in this study did not provide a cutoff value. Second, we extracted multiple trials from one article, which may have increased statistical deviation. Third, due to limited numbers of trials, we did not evaluate the efficacy of miRNA-34a for diagnosis of NASH. Fourth, due to a lack of some data, we did not directly analyze the BMI by meta-regression; Finally, we may have ignored some available literature.

\section{Conclusion}

Based on the above analysis, we conclude that serum miRNA could be considered as a promising non-invasive test for diagnosis of NASH. In particular, serum miRNA may provide effective guidance to clinicians on early diagnosis because of its prominent sensitivity. Moreover, among the well-studied serum miRNAs, miRNA-34a is the most available diagnostic index for NAFLD. It should be noticed that BMI may influence the diagnostic performance, and more research on this aspect is necessary in the future.

\section{Supplementary information}

Supplementary information accompanies this paper at https://doi.org/10. 1186/s12876-020-01334-8

Additional file 1: Figure S1. Quality assessment of the included trials Figure S2. Diagnostic efficacy of serum miRNA-122 for total NAFLD (case vs. control). Figure S3. Diagnostic efficacy of serum miRNA-99a for total NAFLD (case vs. control). Figure S4. Diagnostic efficacy of serum miRNA34a for total NAFLD (case vs. control). Figure S5. Diagnostic efficacy of serum miRNA for NASH (NAS $\geq 5$ vs. < 5). Figure S6. Diagnostic efficacy of serum miRNA for NAFLD (NAFLD vs. healthy control). Figure S7. Diagnostic efficacy of serum miRNA for distinguishing NASH from NAFL.

Table S1. Basic characteristics of the included trials. Table S2. Heterogeneity of serum miRNA for diagnosing total NAFLD after omitting the trials with study factors. PRISMA 2009 Checklist.

\section{Abbreviations}

ALT: Alanine aminotransferase; AST: Aspartate aminotransferase; AUROC: Area under the receiver operating characteristics curve; BMI: Body mass index; Cl: Confidence interval; CK-18: Cytokeratin 18; DOR: Diagnostic odds ratio; FN: False negative; FP: False positive; PLR: Positive likelihood ratio; NAFL: Nonalcoholic fatty liver (simple steatosis); NAFLD: Nonalcoholic fatty liver disease; NAS: NAFLD activity score; NASH: Nonalcoholic steatohepatitis; NLR: Negative likelihood ratio; PPARa: Peroxisome proliferator-activated receptor a; PNPLA3: Patatin like phospholipase domain containing 3; PUMA: p53 up-regulated modulator of apoptosis; QUADAS: Quality assessment of diagnostic accuracy studies; RT-PCR: Reverse transcriptionpolymerase chain reaction; SROC: Summary receiver-operating characteristics; TN: True negative; TP: True positive

\section{Acknowledgements}

In this work, faithfully thanks for professor Xiaoyuan Xu.

\section{Authors' contributions}

SLX: study concept and design, title, abstract, full-text screening, data abstraction, statistical analysis, data interpretation, and drafting the article. QZ, XFC: study design, title, abstract, screening, data interpretation, critical revision of the manuscript. JHX and YYY: data interpretation, revision of the manuscript. All authors read and approved the final manuscript.

\section{Funding}

This study was supported by National Science and Technology Major Project of China. (NO. 2017ZX10202202). The funding body supported the data collection used in this study. The funding body has no role in design of the study, analysis, interpretation of data, preparation of the manuscript and decision to publish.

\section{Availability of data and materials}

The datasets used and/or analyzed during the current study are available from the corresponding authors upon reasonable request.

Ethics approval and consent to participate

Not applicable.

Consent for publication

Not applicable.

\section{Competing interests}

The authors declare that they have no competing interests.

Received: 7 March 2020 Accepted: 3 June 2020

Published online: 12 June 2020

\section{References}

1. Cotter TG, Rinella M. NAFLD 2020: the state of the disease. Gastroenterology. 2020. https://doi.org/10.1053/j.gastro.2020.01.052.

2. Zhou J, Zhou F, Wang W, Zhang XJ, Ji YX, Zhang P, et al. Epidemiological feature of NAFLD from 1999 to 2018 in China. Hepatology. 2020. https://doi. org/10.1002/hep.31150. 
3. Adams LA, Lymp JF, St Sauver J, Sanderson SO, Lindor KD, Feldstein A, et al. The natural history of nonalcoholic fatty liver disease: a population-based cohort study. Gastroenterology. 2005;129(1):113-21.

4. Febbraio MA, Reibe S, Shalapour S, Ooi GJ, Watt MJ, Karin M. Preclinical models for studying NASH-driven HCC: how useful are they? Cell Metab. 2019;29(1):18-26.

5. Baffy G, Brunt EM, Caldwell SH. Hepatocellular carcinoma in non-alcoholic fatty liver disease: an emerging menace. J Hepatol. 2012;56(6):1384-91.

6. Feldstein AE, Wieckowska A, Lopez AR, Liu YC, Zein NN, McCullough AJ. Cytokeratin-18 fragment levels as noninvasive biomarkers for nonalcoholic steatohepatitis: a multicenter validation study. Hepatology. 2009;50(4):1072-8.

7. Cusi K, Chang Z, Harrison S, Lomonaco R, Bril F, Orsak B, et al. Limited value of plasma cytokeratin-18 as a biomarker for NASH and fibrosis in patients with non-alcoholic fatty liver disease. J Hepatol. 2014;60(1):167-74.

8. Castera L, Friedrich-Rust M, Loomba R. Noninvasive assessment of liver disease in patients with nonalcoholic fatty liver disease. Gastroenterology. 2019;156(5):1264-81.

9. Dongiovanni $\mathrm{P}$, Meroni $M$, Longo M, Fargion S, Fracanzani AL. miRNA Signature in NAFLD: A Turning Point for a Non-Invasive Diagnosis. Int J Mol Sci. 2018;19(12):3966

10. Wang L, Zhang N, Wang Z, Ai DM, Cao ZY, Pan HP. Decreased MiR-155 level in the peripheral blood of non-alcoholic fatty liver disease patients may serve as a biomarker and may influence LXR activity. Cell Physiol Biochem. 2016;39(6):2239-48.

11. Ye D, Zhang T, Lou G, Xu W, Dong F, Chen G, et al. Plasma miR-17, miR-20a, miR-20b and miR-122 as potential biomarkers for diagnosis of NAFLD in type 2 diabetes mellitus patients. Life Sci. 2018;208:201-7.

12. Yu F, Wang X, Zhao H, Hao Y, Wang W. Decreased serum miR-1296 may serve as an early biomarker for the diagnosis of non-alcoholic fatty liver disease. Clin Lab. 2019. https://doi.org/10.7754/Clin.Lab.2019.190335.

13. Yu Y, Zhu J, Liu J, Huang M, Wan JX. Identification of 8-miRNAs as biomarkers for nonalcoholic fatty liver disease. J Cell Physiol. 2019;234(10): 17361-9.

14. Liu XL, Cao HX, Fan JG. MicroRNAs as biomarkers and regulators of nonalcoholic fatty liver disease. J Dig Dis. 2016;17(11):708-15.

15. Cai C, Lin Y, Yu C. Circulating miRNAs as novel diagnostic biomarkers in nonalcoholic fatty liver disease: a systematic review and meta-analysis. Can J Gastroenterol Hepatol. 2019. https://doi.org/10.1155/2019/2096161.

16. Xiao G, Zhu S, Xiao X, Yan L, Yang J, Wu G. Comparison of laboratory tests, ultrasound, or magnetic resonance elastography to detect fibrosis in patients with nonalcoholic fatty liver disease: a meta-analysis. Hepatology. 2017;66(5):1486-501.

17. Park CC, Nguyen P, Hernandez C, Bettencourt R, Ramirez K, Fortney L, et al. Magnetic resonance Elastography vs transient Elastography in detection of fibrosis and noninvasive measurement of Steatosis in patients with biopsy-proven nonalcoholic fatty liver disease. Gastroenterology. 2017;152(3):598-607.

18. Pu K, Wang Y, Bai S, Wei H, Zhou Y, Fan J, et al. Diagnostic accuracy of controlled attenuation parameter (CAP) as a non-invasive test for steatosis in suspected non-alcoholic fatty liver disease: a systematic review and metaanalysis. BMC Gastroenterol. 2019;19(1):51.

19. Deeks JJ, Macaskill P, Irwig L. The performance of tests of publication bias and other sample size effects in systematic reviews of diagnostic test accuracy was assessed. J Clin Epidemiol. 2005;58(9):882-93.

20. Auguet T, Aragonès G, Berlanga A, Guiu-Jurado E, Martí A, Martínez S, et al. miR33a/miR33b* and miR122 as Possible Contributors to Hepatic Lipid Metabolism in Obese Women with Nonalcoholic Fatty Liver Disease. Int J Mol Sci. 2016;17(10):1620.

21. Celikbilek M, Baskol M, Taheri S, Deniz K, Dogan S, Zararsiz G, et al. Circulating microRNAs in patients with non-alcoholic fatty liver disease. World J Hepatol. 2014;6(8):613-20.

22. Pirola CJ, Fernández Gianotti T, Castaño GO, Mallardi P, San Martino J, Mora Gonzalez Lopez Ledesma M, et al. Circulating microRNA signature in nonalcoholic fatty liver disease: from serum non-coding RNAs to liver histology and disease pathogenesis. Gut. 2015;64(5):800-12.

23. Hendy OM, Rabie H, El Fouly A, Abdel-Samiee M, Abdelmotelb N, Elshormilisy AA, et al. The circulating micro-RNAs (-122, -34a and -99a) as predictive biomarkers for non-alcoholic fatty liver diseases. Diabetes Metab Syndr Obes. 2019;12:2715-23.

24. López-Riera M, Conde I, Quintas G, Pedrola L, Zaragoza Á, Perez-Rojas J, et al. Non-invasive prediction of NAFLD severity: a comprehensive, independent validation of previously postulated serum microRNA biomarkers. Sci Rep. 2018;8(1):10606.

25. Salvoza NC, Klinzing DC, Gopez-Cervantes J, Baclig MO. Association of Circulating Serum miR-34a and miR-122 with dyslipidemia among patients with non-alcoholic fatty liver disease. PLoS One. 2016;11(4):e0153497.

26. Tan Y, Ge G, Pan T, Wen D, Gan J. A pilot study of serum microRNAs panel as potential biomarkers for diagnosis of nonalcoholic fatty liver disease. PLoS One. 2014;9(8):e105192.

27. Liu XL, Pan Q, Zhang RN, Shen F, Yan SY, Sun C, et al. Disease-specific miR34 a as diagnostic marker of non-alcoholic steatohepatitis in a Chinese population. World J Gastroenterol. 2016;22(44):9844-52.

28. Jampoka K, Muangpaisarn P, Khongnomnan K, Treeprasertsuk S, Tangkijvanich P, Payungporn S. Serum miR-29a and miR-122 as potential biomarkers for non-alcoholic fatty liver disease (NAFLD). Microrna. 2018;7(3): 215-22.

29. Younossi ZM, Koenig AB, Abdelatif D, Fazel Y, Henry L, Wymer M, et al. Global epidemiology of nonalcoholic fatty liver disease-meta-analytic assessment of prevalence, incidence, and outcomes. Hepatology. 2016;64(1): 73-84.

30. Barbois S, Arvieux C, Leroy V, Reche F, Stürm N, Borel AL. Benefit-risk of intraoperative liver biopsy during bariatric surgery: review and perspectives. Surg Obes Relat Dis. 2017;13(10):1780-6.

31. Mathiesen UL, Franzén LE, Frydén A, Foberg U, Bodemar G. The clinical significance of slightly to moderately increased liver transaminase values in asymptomatic patients. Scand J Gastroenterol. 1999;34(1):85-91.

32. Kwok R, Tse YK, Wong GL, Ha Y, Lee AU, Ngu MC, et al. Systematic review with meta-analysis: non-invasive assessment of non-alcoholic fatty liver disease--the role of transient elastography and plasma cytokeratin-18 fragments. Aliment Pharmacol Ther. 2014;39(3):254-69.

33. Musso G, Gambino R, Cassader M, Pagano G. Meta-analysis: natural history of non-alcoholic fatty liver disease (NAFLD) and diagnostic accuracy of noninvasive tests for liver disease severity. Ann Med. 2011;43(8):617-49.

34. Tamimi TI, Elgouhari HM, Alkhouri N, Yerian LM, Berk MP, Lopez R, et al. An apoptosis panel for nonalcoholic steatohepatitis diagnosis. J Hepatol. 2011; 54(6):1224-9.

35. Huang JF, Yeh ML, Huang CF, Huang Cl, Tsai PC, Tai CM, et al. Cytokeratin18 and uric acid predicts disease severity in Taiwanese nonalcoholic steatohepatitis patients. PLoS One. 2017. https://doi.org/10.1371/journal. pone.0174394.

36. Fielding CA, Jones GW, McLoughlin RM, McLeod L, Hammond VJ, Uceda J، Williams AS, et al. Interleukin-6 signaling drives fibrosis in unresolved inflammation. Immunity. 2014;40(1):40-50.

37. Bril F, McPhaul MJ, Caulfield MP, Castille JM, Poynard T, Soldevila-Pico C, et al. Performance of the SteatoTest, ActiTest, NashTest and FibroTest in a multiethnic cohort of patients with type 2 diabetes mellitus. J Investig Med. 2019;67(2):303-11.

38. Liu XL, Pan Q, Cao HX, Xin FZ, Zhao ZH, Yang RX, et al. Lipotoxic hepatocytederived Exosomal miR-192-5p activates macrophages via Rictor/Akt/FoxO1 signaling in NAFLD. Hepatology. 2019. https://doi.org/10.1002/hep.31050.

39. Gan M, Shen L, Fan Y, Tan Y, Zheng T, Tang G, et al. MicroRNA-451 and Genistein Ameliorate Nonalcoholic Steatohepatitis in Mice. Int J Mol Sci. 2019;20(23):6084.

40. Klieser E, Mayr C, Kiesslich T, Wissniowski T, Fazio PD, Neureiter D, et al. The Crosstalk of miRNA and Oxidative Stress in the Liver: From Physiology to Pathology and Clinical Implications. Int J Mol Sci. 2019;20(21):5266.

41. Gjorgjieva M, Sobolewski C, Dolicka D, Correia de Sousa M, Foti M. miRNAs and NAFLD: from pathophysiology to therapy. Gut. 2019;68(11):2065-79.

42. Becker PP, Rau M, Schmitt J, Malsch C, Hammer C, Bantel H, et al. Performance of serum microRNAs $-122,-192$ and -21 as biomarkers in patients with non-alcoholic Steatohepatitis. PLoS One. 2015. https://doi.org/ 10.1371/journal.pone.0142661.

43. Erhartova D, Cahova M, Dankova H, Heczkova M, Mikova I, Sticova E, et al. Serum miR-33a is associated with steatosis and inflammation in patients with non-alcoholic fatty liver disease after liver transplantation. PLoS One. 2019. https://doi.org/10.1371/journal.pone.0224820.

44. Liu CH, Ampuero J, Gil-Gómez A, Montero-Vallejo R, Rojas Á, MuñozHernández R, et al. miRNAs in patients with non-alcoholic fatty liver disease: a systematic review and meta-analysis. J Hepatol. 2018;69(6):1335-48.

45. Torres JL, Novo-Veleiro I, Manzanedo L, Alvela-Suárez L, Macías R, Laso FJ, et al. Role of microRNAs in alcohol-induced liver disorders and nonalcoholic fatty liver disease. World J Gastroenterol. 2018;24(36):4104-18. 
46. Ding J, Li M, Wan X, Jin X, Chen S, Yu C, et al. Effect of miR-34a in regulating steatosis by targeting PPARa expression in nonalcoholic fatty liver disease. Sci Rep. 2015;5:13729.

47. Ip E, Farrell GC, Robertson G, Hall P, Kirsch R, Leclercq I. Central role of PPARalpha-dependent hepatic lipid turnover in dietary steatohepatitis in mice. Hepatology. 2003;38(1):123-32.

48. Castro RE, Ferreira DM, Afonso MB, Borralho PM, Machado MV, Cortez-Pinto $\mathrm{H}$, et al. miR-34a/SIRT1/p53 is suppressed by ursodeoxycholic acid in the rat liver and activated by disease severity in human non-alcoholic fatty liver disease. J Hepatol. 2013;58(1):119-25.

49. Fan JG, Kim SU, Wong W. New trends on obesity and NAFLD in Asia. J Hepatol. 2017;67(4):862-73.

50. Romeo S, Kozlitina J, Xing C, Pertsemlidis A, Cox D, Pennacchio LA, et al. Genetic variation in PNPLA3 confers susceptibility to nonalcoholic fatty liver disease. Nat Genet. 2008:40(12):1461-5.

51. Li Y, Xing C, Cohen JC, Hobbs HH. Genetic variant in PNPLA3 is associated with nonalcoholic fatty liver disease in China. Hepatology. 2012;55(1):327-8.

\section{Publisher's Note}

Springer Nature remains neutral with regard to jurisdictional claims in published maps and institutional affiliations.

Ready to submit your research? Choose BMC and benefit from:

- fast, convenient online submission

- thorough peer review by experienced researchers in your field

- rapid publication on acceptance

- support for research data, including large and complex data types

- gold Open Access which fosters wider collaboration and increased citations

- maximum visibility for your research: over $100 \mathrm{M}$ website views per year

At BMC, research is always in progress.

Learn more biomedcentral.com/submissions 